\title{
The development of a new continuous dyeing method based on an aerosol delivery system
}

DOI:

10.1111/j.1478-4408.1999.tb00321.x

\section{Document Version}

Accepted author manuscript

Link to publication record in Manchester Research Explorer

\section{Citation for published version (APA):}

Cork, C., Foster, P., Oulton, D., \& Chowdhury, M. (1999). The development of a new continuous dyeing method based on an aerosol delivery system. Coloration Technology, 115(11), 333-338. https://doi.org/10.1111/j.14784408.1999.tb00321.x

\section{Published in:}

Coloration Technology

\section{Citing this paper}

Please note that where the full-text provided on Manchester Research Explorer is the Author Accepted Manuscript or Proof version this may differ from the final Published version. If citing, it is advised that you check and use the publisher's definitive version.

\section{General rights}

Copyright and moral rights for the publications made accessible in the Research Explorer are retained by the authors and/or other copyright owners and it is a condition of accessing publications that users recognise and abide by the legal requirements associated with these rights.

\section{Takedown policy}

If you believe that this document breaches copyright please refer to the University of Manchester's Takedown Procedures [http://man.ac.uk/04Y6Bo] or contact uml.scholarlycommunications@manchester.ac.uk providing relevant details, so we can investigate your claim.

\section{OPEN ACCESS}




\title{
The development of a new continuous dyeing method based on an aerosol delivery system
}

\section{R Cork, P W Foster, D P Oulton and M D H Chowdhury}

\author{
Dept of Textiles, UMIST, PO Box 88, Manchester M60 1QD, UK
}

\begin{abstract}
A continuous method of dyeing polyester yarn has been developed in which yarn is forced into a moving plug within apparatus based on the Fibre-M texturing system. Dye is then applied in droplet form to the yarn plug. The method relies on the dispersion of low-molecular-weight disperse dyes in a solvent medium to provide a uniform application to the core of the plug. The demands of the textile industry require an extension of the method to a wider range of dye types in a water-based medium. This paper describes a feasibility study of an aerosol method of dye application to a yarn plug.
\end{abstract}

\section{INTRODUCTION}

The development of clean technology is of increasing importance. In the textile and clothing industry, expensive clean-up plants have to be installed at dyehouses before the large volumes of water involved in dyeing can be returned to the river system. Current dyehouse practices do not meet, or only meet with great difficulty, the legal requirements for effluent discharges. Much discussion has focused on these environmental problems [1-4]. Often solutions can be developed from the point of view of deactivating or removing pollutants after they have been produced [5-7]. However, there has long been pressure to develop new, environmentally friendly dyeing methods.

One promising environmentally friendly development in recent years has been the use of supercritical fluids, particularly carbon dioxide, as a dyeing medium $[8,9]$. The method is currently limited to a batch process, but a continuous system is more desirable for economic reasons. One promising environmentally friendly development in recent years has been the use of supercritical fluids, particularly carbon dioxide, as a dyeing medium $[8,9]$. The method is currently limited to a batch process, but a continuous system is more desirable for economic reasons. Of particular note in continuous dyeing have been:

(a) A method of dyeing and bulking acrylic yarns [10]

(b) A method of padding dye on yarn using compressedair cells to impregnate and then squeeze the dyed yarn [11]

(c) A continuous method of dyeing yarn or similar material by spray application of dye [12]

(d) A system for texturing and dyeing yarn using hot fluid flow into a yarn that has been stacked and compressed into a confined space by steam pressure [13].

A well established continuous method is to produce wetspun fibres to which colorants have been added to the swollen 'never dried' fibre structure. Compared with other dyeing methods, this gel dyeing technique is environmentally friendly. Mass-pigmented products are also used but have only found small market niches where the colour fastness of the pigment, relative to that of a dye, outweighs the disadvantages. A major problem with gel dyeing is that dedicated equipment specific to each colour is required. Accordingly, even the largest manufacturer can only produce a few basic colours. In addition, dyeing occurs early in the transformation of a fibre to a garment, whereas it is best to add value at the later stage of any process in order to minimise costs. Furthermore, the process is less flexible in that larger quantities of colours have to be made months before the market demands are known. Accordingly, while spun-dyed fabrics have a place (e.g. for high light fastness in automobile fabrics), it is a limited one.

At UMIST, Chowdhury has developed a continuous effluent-free dyeing process for yarn [14], based on the Fibre-M Constant Bulk Texturing system [15]. A schematic diagram of the equipment is shown in Figure 1 . The yarn enters the system via a labyrinth seal (A). Pressurised superheated steam is fed in at B and the yarn is jet textured at $C$. While the yarn is still hot and plastic, a yarn plug is created at $\mathrm{D}$ and moves upwards within a yarn reservoir (or dwell tube) between D and G. At the point just above where the plug is formed, dyes in organic solvent solution is injected (E). Chowdhury's process is attractive, in that while jet texturing of polyester may be carried out at very high speeds (up to $6500 \mathrm{~m} / \mathrm{min}$ ), the yarn within the reservoir is slow moving and potentially complicated sequences of yarn treatments can be carried out. Before tension is reapplied to the hot yarn, it is necessary to cool it to below the second-order transition temperature. This is achieved by sucking air through the yarn reservoir at $\mathrm{F}$. The high boiling point solvent is then condensed out of the warm cooling air.

Chowdhury has shown that a technically satisfactory product with good dye migration can be achieved without any dyes or solvent entering any watercourse or smokestack. The solvent is essential in order to produce a uniform dispersion of dye into the heart of the yarn plug. 


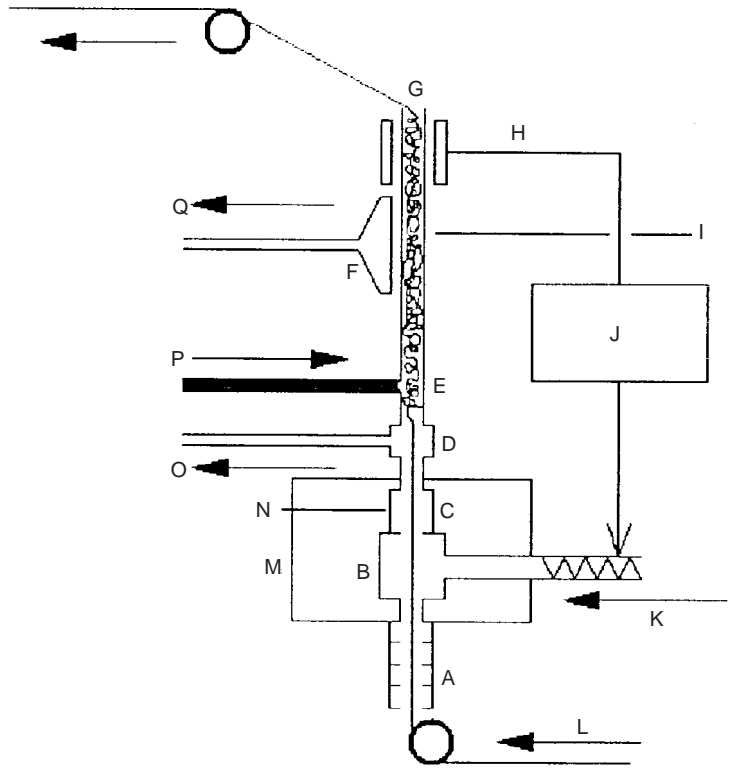

$\begin{array}{ll}\text { A Labyrinth seal } & \text { J Feedback loop to heater } \\ \text { B Pressurised steam feed } & \text { K Steam inlet } \\ \text { C Jet texturing } & \text { L Yarn motion } \\ \text { D Yarn plug production } & \text { M Manifold } \\ \text { E Dwell tube start } & \text { N Plug former and texturing jet } \\ \text { F Air suction } & \text { O Steam outlet } \\ \text { G Dwell tube end } & \text { P Dye injection } \\ \text { H Photodetectors } & \text { Q Air suction } \\ \text { I Yarn reservoir } & \end{array}$

Figure 1 The UMIST system for continuous dyeing of yarn

However, it would be more desirable to replace the solvent with a water-based system. With a water-based system it is possible to extend the range of dyes that could be employed.

This paper outlines progress towards a method of replacing the solvent with water using an aerosol delivery system to deliver dye directly to fibres within a yarn plug.

\section{EXPERIMENTAL}

\section{Aerosol source}

The basic theory of the absorption of aerosols by fibrous assemblies is well known [16-18]. There are three main mechanisms for the deposition of particles on the fibre surface. These are direct interception, inertial deposition and diffusion due to Brownian motion. Direct interception depends on the droplet and fibre geometries. The effect decreases with droplet diameter. The inertial component depends on the mass and velocity of the droplet and thus indirectly on the droplet diameter. The effect of this also decreases with droplet diameter. In contrast very small droplets are more susceptible to impact with fibres due to the agitation caused by Brownian motion. Overall, aerosol penetration increases with a reduction in droplet diameter but there are reasons for expecting a decrease in penetration at very small diameters. However, a precise quantitative theory has not been established. Empirically, there is general agreement that penetration increases down to the sub-micron range [18]. However, there is disagreement as to the existence of a maximum penetrating diameter. This is not unexpected given the complex nature of the problem and the large number of parameters for any aerosol/fibre system. In practice, all available commercial aerosol devices gave droplet sizes that were greater than the sub-micron range.

The application of dye using sprays is not new [11]. However, in the current work, initial trials based on paint sprays and industrial nebulisers showed that penetration of fibre masses was poor. Therefore, aerosols were sought that could deliver a smaller droplet size. Trials began on a Sidestream nebuliser manufactured by Medic-Aid (W Sussex, UK). The so-called Sidestream nebuliser was originally designed to deliver medicinal drugs to patients in aerosol form. According to the suppliers, it produces an aerosol where over $80 \%$ of the particles are under $5 \mu \mathrm{m}$ in diameter. Although some of the aerosol was very fine and would evaporate in air a few centimetres from the nebuliser, some larger droplets were observed to be ejected from the nebuliser at intervals. These were removed by a gravity filter consisting of a section of vertical tubing between the aerosol and the yarn plug. In this, heavy droplets are less effected by the upward flow and fall back to the nebuliser. In contrast lighter particles would be carried to the top of the tube.

At the operating pressure of between 1 and 1.4 bar the air flow was approximately $6 \mathrm{l} / \mathrm{min}$. The manufacturer quotes an aerosol delivery rate of $0.4 \mathrm{ml} / \mathrm{min}$. Trials were conducted with liquid Dispersol C-R dye, supplied by Zeneca, at a dilution of one part Dispersol C-R liquid to two parts water. Despite the viscous nature of the concentrated dye, delivery rates equivalent to those quoted by the manufacturer were obtained experimentally (Figure 2). The viscous nature of the dye solution did not appear to cause blockages in the system. After use, the nebuliser was rinsed in cold water and no reduction in performance was noted during the course of the test programme.

Given the maximum delivery rate, it is possible to calculate the maximum theoretical mass of yarn that can be dyed in a given time using one nebuliser. Assuming a dye add-on level of $3 \%$, the theoretical maximum proces-

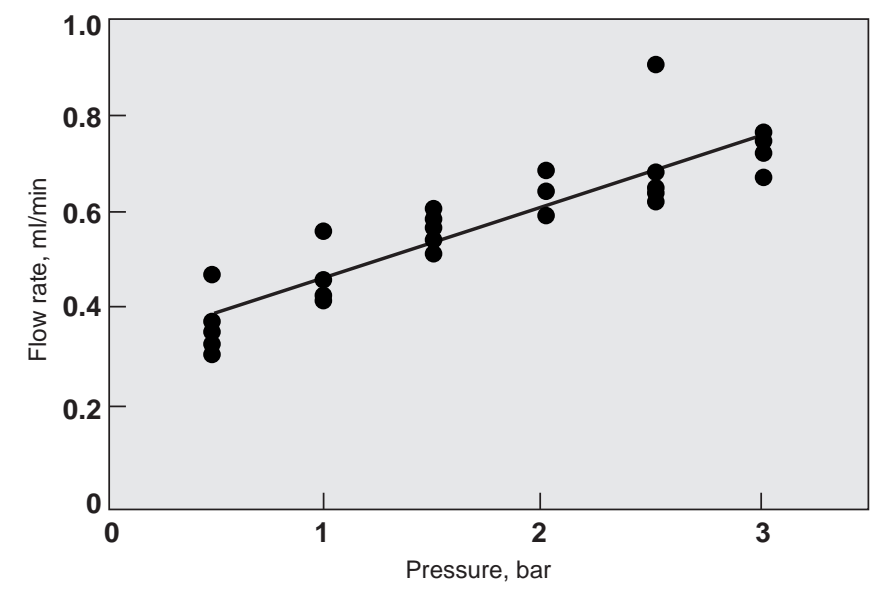

Figure 2 Variation of aerosol flow with air input pressure 
sing rate would be $14 \mathrm{~g}$ per min. For a 15 tex yarn this corresponds to a dyeing rate of $1000 \mathrm{~m}$ of yarn per min. This is the correct order of magnitude for commercial production. However, for higher processing speeds, multistation nebulisers would be required.

Trials were performed on two experimental applicators employing the nebuliser. In initial tests, dye was applied to a hand-formed plug. When these trials proved successful, a more complex system for testing the application to a Fibre-M plug was designed.

There are potential health problems associated with the use of aerosols. However, it is envisaged that, in a production version of the applicator, the aerosol will be totally contained within the system. Nevertheless, for reasons of safety during the research phase, all tests were performed in a fume cupboard on a stationary plug.

\section{Hand-formed plug}

Figure 3 shows the experimental apparatus. A section of Perspex tubing fitted over the nebuliser acted both as the sample holder and the gravity filter. A plug of 167 dtex polyester yarn was fitted to the top of the tube and dye was applied for $10 \mathrm{~min}$. The penetration of dye could be observed through the Perspex tube.

\section{Fibre-M plug}

A schematic of the Fibre-M applicator is shown in Figure 4. Aerosol is now injected from the periphery of the yarn plug in order to facilitate plug movement in a commercial system. Early trials indicated that aerosol flow to the plug was less than for the hand-formed plug. It was assumed that restricted flow might be a result of inhibited aerosol production. Therefore a valve was introduced whereby aerosol was created and vented for $5 \mathrm{~s}$. Next the valve was

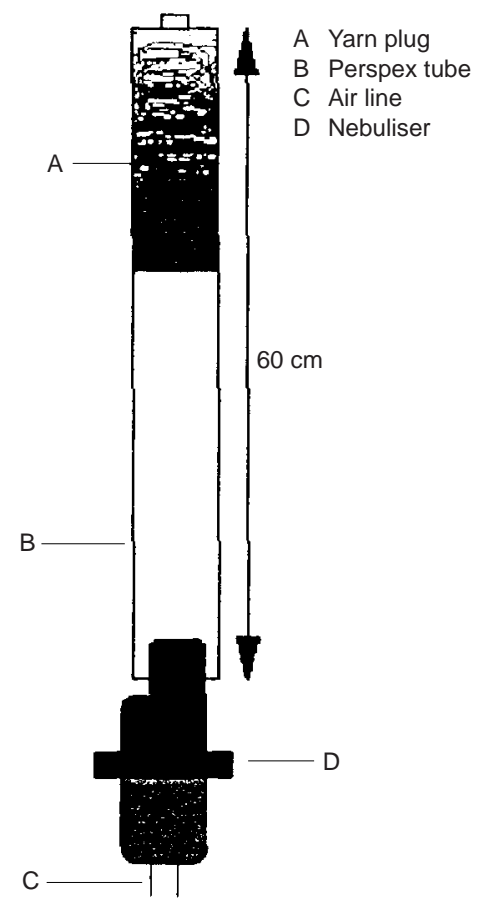

Figure 3 Applicator for hand formed plug

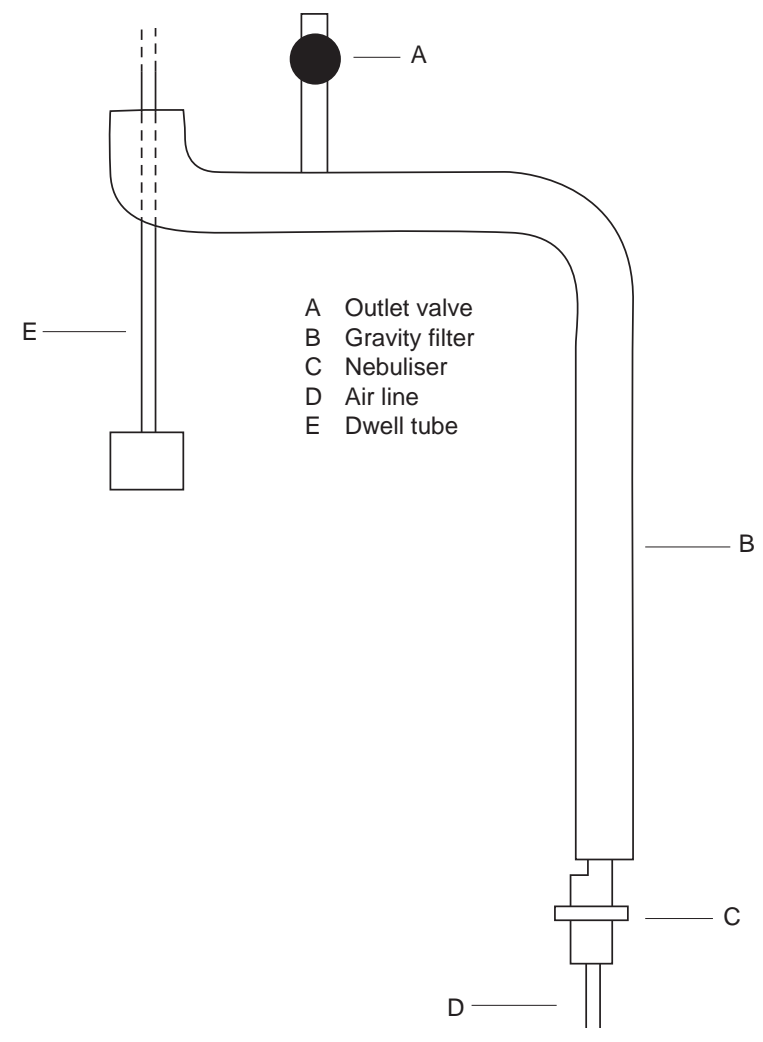

Figure 4 Applicator for the Fibre-M plug

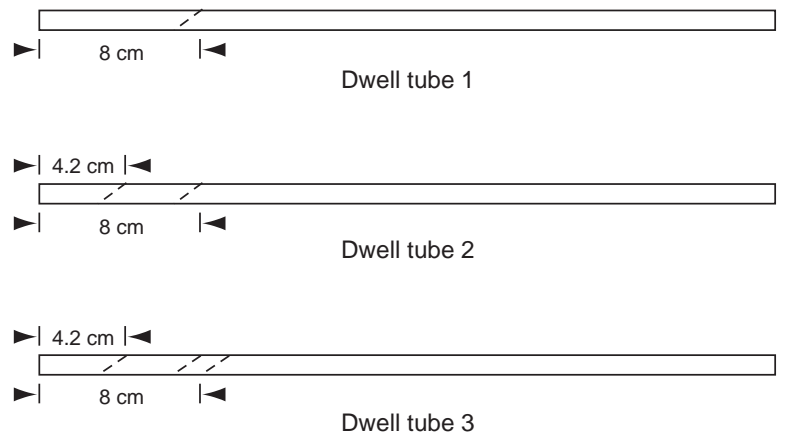

Figure 5 Design of dwell tubes

closed for $5 \mathrm{~s}$ to duct the aerosol through the dwell tube. The cycle was then repeated.

For trials designed to improve plug penetration, three dwell tubes (1,2 and 3) were constructed with different configurations (Figure 5). Dwell tube 1 has three inlet slots located $8 \mathrm{~cm}$ from one end of the tube. Surplus aerosol is vented from the ends of the tube. Dwell tube 2 has additional vents at a distance of approximately $4 \mathrm{~cm}$ from the inlets. In dwell tube 3 is similar to tube 2 but has an additional set of inlet slots.

After application of aerosol dye, the plug was removed from the dwell tube and fixed in an oven at $190{ }^{\circ} \mathrm{C}$ for 30 min, sliced into numbered sections and weighed. Knowing the original plug length and the total mass of the plug it was possible to calculate the distance of each segment along the dwell tube when in situ. Digital images of plug crosssections were obtained using a video microscope with an 
SM camera (version 1.0.4) software. Image analysis was used to assess the degree of dye penetration to the core of each section and subsequently the spread of dye through the sections. For this purpose an algorithm was developed for implementation with Visilog image analysis software.

Different levels of application to the plug produced various shades from light blue to black. For simplicity, each cross-section was analysed in terms of two levels of dye application:

(a) The percentage of whole plug cross-sectional area that was black

(b) The percentage that was above a certain threshold level of blue.

In order to assess the degree of dye penetration to the core, a similar analysis of the central region of the crosssection was performed.

\section{RESULTS}

\section{Hand-formed plug}

As the air supply to the aerosol was turned on, the dye quickly penetrated the plug and spread up the column. The plug height was $14 \mathrm{~cm}$ when compressed into the apparatus, but extended to just under $30 \mathrm{~cm}$ on removal from the tube. As the plug was not stable on removal from the dwell tube, it was not possible to determine exactly the parts that corresponded with the compressed plug, and thus precise quantification of penetration with image

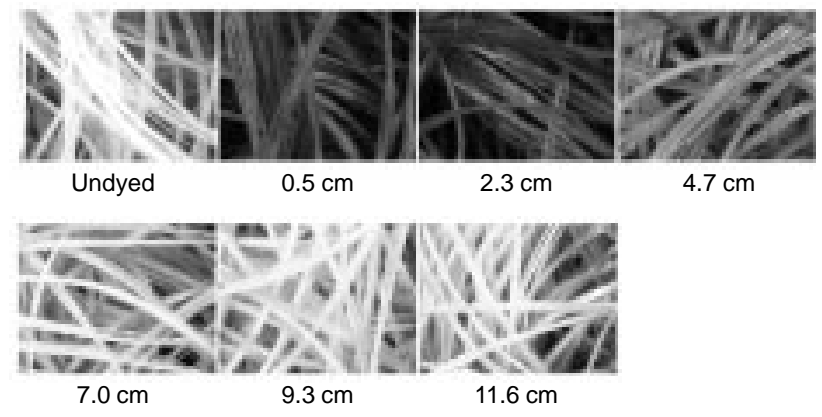

Figure 6 Spread of dye along the hand-formed plug

analysis was not attempted. However, as a guide to the effectiveness of application, images of fibres along the length of the plug, both before and after fixing, were compared with undyed yarn. As expected, dye application was greater at the base of the static plug near the aerosol. In any commercial system developed from the work the configuration would be different; the plug would move past the point of aerosol injection and every cross-section of the plug would have the same exposure to dye. The dye distribution in the static plug gives an estimate of the penetration of dye to the core and the smoothing effect of aerosol penetration away from the point of injection. In the case of the static plug, dye application was achieved throughout the length of the plug with good levels of dye uptake up to and including a position $4.7 \mathrm{~cm}$ from the nebuliser in the plug in situ (Figure 6). There was some dye penetration to $11.6 \mathrm{~cm}$. The same patterns were seen in the yarn before fixing.

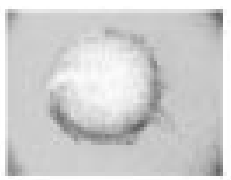

(a)

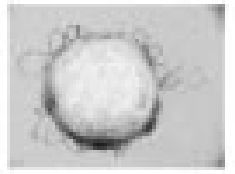

(f)

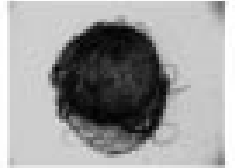

(k)

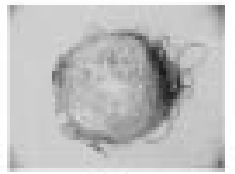

(p)

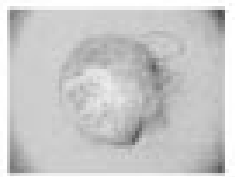

(u)

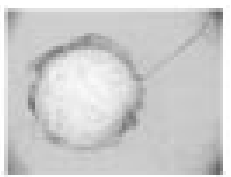

(b)

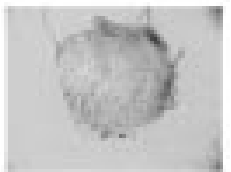

(g)

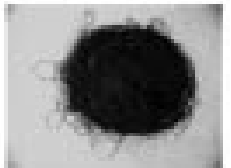

(l)

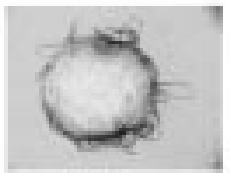

(q)

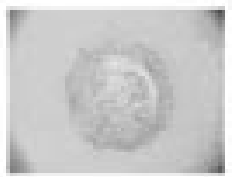

(v)

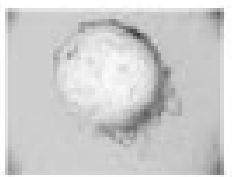

(c)

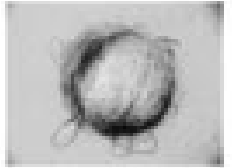

(h)

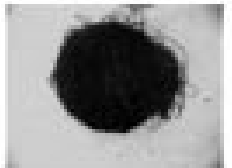

(m)

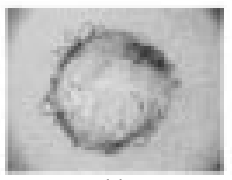

(r)

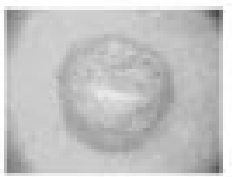

(w)

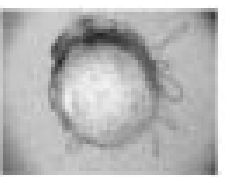

(d)

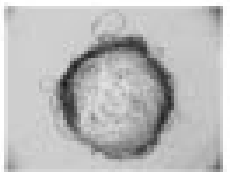

(i)

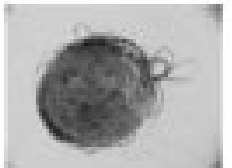

(n)

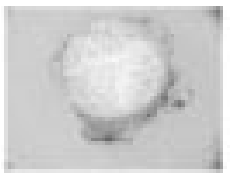

(s)

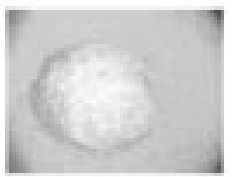

(x)

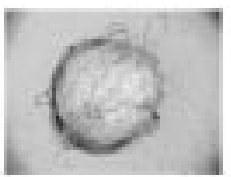

(e)

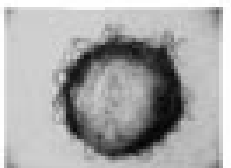

(j)

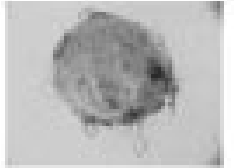

(o)

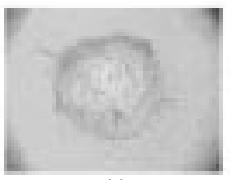

(t)

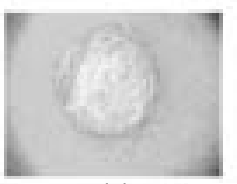

(y)

Figure 7 Series of cross-sections from the Fibre-M plug 


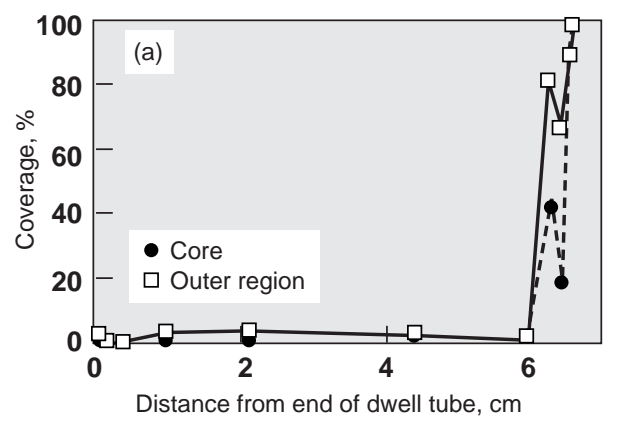

(a) Dwell tube 1

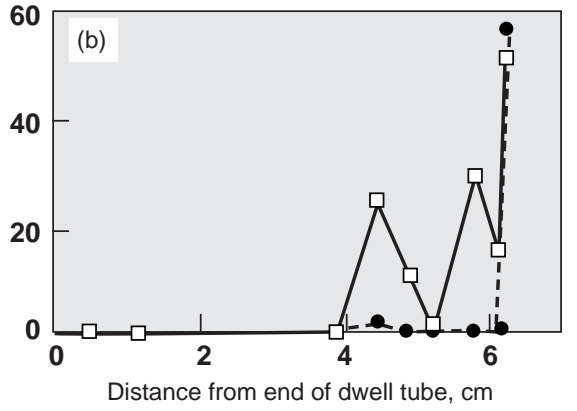

(b) Dwell tube 2

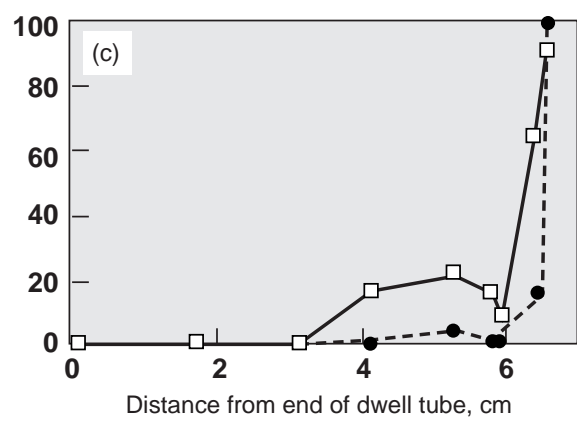

(c) Dwell tube 3

Figure 8 Black threshold coverage for three dwell tubes

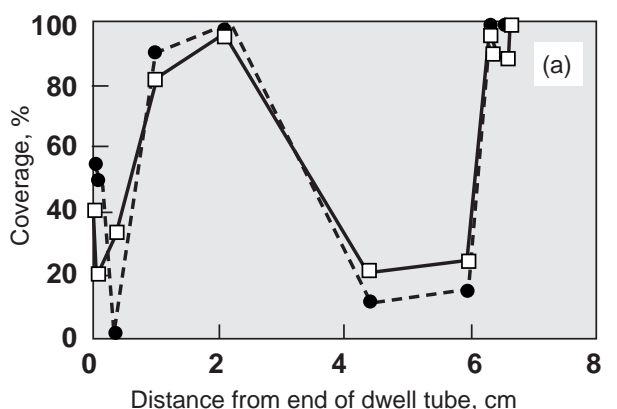

(a) Dwell tube 1
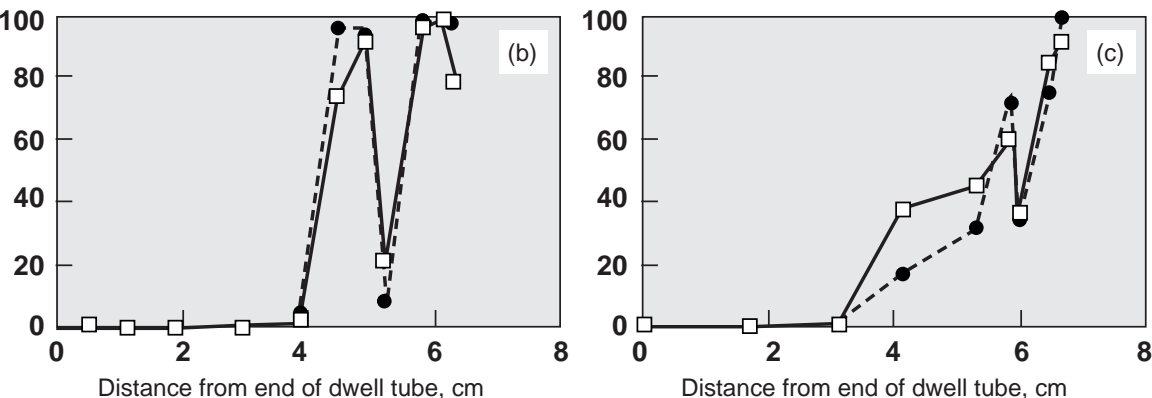

(b) Dwell tube 2

(c) Dwell tube 3

Figure 9 Blue threshold coverage for different dwell tubes (for key see Figure 8)

Overall an application of $1 \%$ was achieved in 15 min on $500 \mathrm{~m}$ of 28 tex yarn. This is lower than the theoretical level of application, probably because the restriction of flow resulted in a reduction in aerosol efficiency.

\section{Fibre-M plug}

In contrast to the hand-formed plug, the Fibre-M plug was stable on removal from the dwell tube and it was possible to determine the exact position of each part when in the applicator. A typical series of plug cross-sections is shown in Figure 7. The black threshold and the blue threshold results of the image analysis of plugs taken from each of the three dwell tubes are shown in Figures 8 and 9 respectively. The results for the blue threshold represent a lower level of dye application than that of the black threshold. Necessarily, the former shows a greater degree of both radial and longitudinal penetration.

For dwell tube 1 the application of the high levels of dye were largely confined to the region of the input slots (Figure 8) at approximately 6-7 $\mathrm{cm}$ from the end of the tube. The addition of extra slots dramatically increased application, but this is largely confined to the outer regions of the plug.

The distribution of dye as measured by the blue threshold shows a greater similarity between the core and the outer regions. The areas dyed are more extensive. For example dwell tube 1 gave high levels right up to the region $1 \mathrm{~cm}$ from the end. Hence the result is anomalous in that lower levels are to be found nearer the slots. It is suggested that this might be a result of dye tunnelling through holes in the plug. Alternatively it is possible that the plug acts as an additional filter of aerosol particles and that those reaching the end are finer and more penetrating. The most consistent distribution is shown by dwell tube 3 (Figure 9c).

It was clear from a qualitative assessment that the application was less than for the hand-formed plug. The tests with hand formed plugs differs from the tests with the Fibre-M plug in terms of the plug density and dwell tube diameter. The packing factor for the hand-formed plug is only $4 \%$ compared with $28.6 \%$ for the Fibre-M plug (Table 1). In addition the Perspex tube has a much larger diameter than the copper dwell tube used for the Fibre-M plug.

Possible ways to improve application are to decrease the Fibre-M plug density or to produce a continuous equivalent of the hand-formed plug, perhaps by the use of a stuffer box, i.e. a chamber where yarn is forced in by mechanical means.

\section{Removal of residual aerosol effluent}

The proposed aerosol system raises obvious questions 


\begin{tabular}{|c|c|c|c|c|c|c|c|c|c|}
\hline & Fibre & $\begin{array}{l}\text { Yarn } \\
\text { linear } \\
\text { density (tex) }\end{array}$ & $\begin{array}{l}\text { Plug } \\
\text { length } \\
\text { (cm) }\end{array}$ & $\begin{array}{l}\text { Plug } \\
\text { diameter } \\
\text { (cm) }\end{array}$ & $\begin{array}{l}\text { Plug } \\
\text { mass } \\
(\mathrm{g})\end{array}$ & $\begin{array}{l}\text { Yarn } \\
\text { length } \\
\text { in plug }(m)\end{array}$ & $\begin{array}{l}\text { Plug } \\
\text { density } \\
(\mathrm{g} / \mathrm{ml})\end{array}$ & $\begin{array}{l}\text { Polyester } \\
\text { density } \\
(\mathrm{g} / \mathrm{ml})\end{array}$ & $\begin{array}{l}\text { Packing } \\
\text { factor } \\
(\%)\end{array}$ \\
\hline Fibre-M plug & Polyester & 16.7 & 75.0 & 0.37 & 3.26 & 195 & 0.395 & 1.38 & 28.6 \\
\hline Hand-formed plug & Polyester & 16.7 & 13.5 & 3.81 & 8.45 & 506 & 0.055 & 1.38 & 4.0 \\
\hline
\end{tabular}

about the handling of any aerosol waste in order to eliminate any possible hazardous releases into the environment. It would be undesirable to eliminate waterborne effluent only to create gaseous pollution. However, for the proposed aerosol-based system the problem of atmospheric pollution is manageable. Firstly, a commercial applicator could be made to operate as a closed system, eliminating all gaseous discharge. If venting from the tube is required to increase flow, this could be recycled to the aerosol input. However, if some venting from the system is necessary, there exists a well established technology for removal of aerosols. Commonly used methods are gravitational settling, wet scrubbers, centrifugal separators, electrostatic precipitation and fibrous filters. The latter are the most reliable, efficient and economic [16,17], and of course the aerosol applicator itself performs as a fibrous filter. Nevertheless, if a final stage of filtration is required then one (or a combination) of the methods for aerosol removal could be used. The wet scrubbers would be undesirable as the resulting dye would be in dilute waterbased form. In addition gravitational settling is extremely inefficient at the $5 \mu \mathrm{m}$ range or below [19]. However, centrifugal separators or electrostatic precipitation would produce dye waste in a concentrated liquid form for disposal other than in the river system. A supplementary fibrous filtration system would produce only solid waste.

\section{CONCLUSIONS}

The work has demonstrated the possibility of adapting a medical aerosol system to deliver dye to a yarn plug. The concept is new and, in contrast to spray application methods, produces a significantly different dynamic interaction between dye and fibre during penetration. It has been demonstrated that the aerosol applicator can function with a concentrated dye solution without clogging. Initial tests with a hand-formed plug show that the fine aerosol produced by the applicator can readily penetrate the fibrous assemblies. The level of penetration to the denser Fibre-M plug was less than for the hand- formed plug. However, the results demonstrated are an improvement over the original UMIST Fibre-M dyeing system whereby direct application could not be achieved at the core of the plug.

Further development is required and considerable difficulties have still to be overcome in order to achieve a uniform application of colour in a commercial system. Possible solutions are to improve aerosol flow to the FibreM plug, perhaps by modifications to the plug density or by using an alternative plug formation strategy, for example by the use of a stuffer box.

It is believed that the fine aerosol system could have commercial implications in fields other than dyeing. For example, the system could also be used for the application of finishes and other surface treatments to both yarns and fabrics.

\section{REFERENCES}

1. P Cooper, J.S.D.C., 107 (1991) 384

2. E Syson, J.S.D.C., 107 (1991) 390.

3. B D'Arcy, J.S.D.C., 107 (1991) 387.

4. B G Hazel, J.S.D.C., 107 (1991) 392

5. R Yu-Li Yeh, J. Chem. Technol. Biotechnol., 63 (1995) 48.

6. R Yu-Li Yeh, J. Chem. Technol, Biotechnol., 63 (1995) 55.

7. W S Hickman, J.S.D.C., 109 (1993) 32.

8. W Saus, D Knittel and E Schollmeyer, Text. Res. J., (1993), 63 (3) 135

9. D Knittel and E Schollmeyer, Int. J. Clothing Sci. Tech., 7 (1) (1995) 36.

10. I Hardalov and J Mikhaylova, J.S.D.C., 109 (1993) 369.

11. J Niederhauser, I Devaux and A Morelle, J.S.D.C., 83 (1973) 533.

12. Vepa AG, USP 3835671 (1972).

13. Rhöne Poulenc, BP 1312579 (1973).

14. M D H Chowdhury, PhD thesis, UMIST (1992).

15. PW Foster, Text. Inst. Ind., (1975) 172.

16. G M Hidy, Aerosols (London: Academic Press, 1984).

17. D T Shaw, Fundamentals of aerosol science, (New York: John Wiley, 1978).

18. Aerosol science, Ed. C N Davies (London: Academic Press, 1966).

19. L Theodore and A J Bounicore, Industrial air pollution control equipment for particulates (Cleveland, Ohio: CRC Press, 1976). 